\title{
Clinical feasibility of duct-to-mucosa pancreaticojejunostomy in laparoscopic pancreaticoduodenectomy: Comparison with the same technique in open pancreaticoduodenectomy
}

\author{
Jaryung Han, Young Seok HAN*
}

Division of Hepatobiliary Pancreas Surgery and Liver Transplantation, Department of Surgery, Kyungpook National University Hospital, Daegu, Korea

Introduction: With advances of laparoscopic surgery, laparoscopic pancreaticoduodenectomy (L-PD) was adopted by a few experienced surgeons. Characteristics of PD have been an obstacle to the adoption of L-PD. Duct-to-mucosa pancreaticojejunostomy (DM-PJ) is one of the most commonly used techniques in PD. However, DM-PJ can be considered technically a challenge procedure in L-PD, especially in cases with small pancreatic duct. Therefore, we will present the clinical feasibility and technical tips of DM-PJ in L-PD.

Methods: Between January 2016 and December 2020, we performed 160 cases of PD. Among them, 71 patients underwent totally L-PD. We retrospectively reviewed the medical records to ascertain the safety and the reproducibility of DM-PJ in L-PD and compared DM-PJ in open cases.

Results: Significant pancreatic fistula (International Study Group on Pancreatic Fistula grade B or C) in L-PD was less than 10\%, and this complication was even more higher in open DM-PJ group. The postoperative mortality in L-PD was nil and this result was also lower than open DM-PJ. The postoperative complication higher than Clavien-Dindo classification grade III was identified in 4 patients in L-PD, but recovered by intervention. The conversion to open PD was required in one patient with tumor invasion to the superior mesenteric vein, but there was no open conversion because of technical difficulty of DM-PJ.

Conclusions: DM-PJ in L-PD can be the standard method as in open PD, because postoperative morbidity and mortality were not different significantly. Technical challenging due to characteristics of L-PD will be improved as the laparoscopic surgical techniques and instruments advance. 